Pacific Journal of Mathematics

SUFFICIENT CONDITIONS FOR AN OPTIMAL CONTROL
PROBLEM IN THE CALCULUS OF VARIATIONS 


\section{SUFFICIENT CONDITIONS FOR AN OPTIMAL CONTROL PROBLEM IN THE CALCULUS OF VARIATIONS}

\section{EDwIN H. MookInI}

An arc $C$ is a collection of parameters $b^{\rho}(\rho=1, \cdots, r)$ on an open set $B$ and sets of functions $y^{i}(x), a^{h}(x)(i=1, \cdots, n$; $h=1, \cdots, m)$ defined on an interval $x^{1} \leqq x \leqq x^{2}$ with $y^{i}(x)$ continuous and $\dot{y}^{i}(x), a^{h}(x)$ piecewise continuous. The arc is admissible if it satisfies the differential equations

$$
\dot{y}^{i}=P^{i}(x, y, a) \quad(i=1, \cdots, n)
$$

on $x^{1} \leqq x \leqq x^{2}$ and the end conditions

$$
x^{s}=X^{s}(b), y^{i}\left(x^{s}\right)=Y^{i s}(b) \quad(s=1,2) .
$$

The dot denotes differentiation with respect to $x$. The problem at hand is to find in a class of admissible arcs $C$, an arc $C_{0}$, which minimizes the integral

$$
I(C)=g(b)+\int_{x^{1}}^{x^{2}} f(x, y, a) d x
$$

where $P(x, y, a)$ and $f(x, y, a)$ are assumed to be class $C^{\prime \prime}$ for $(x, y, a)$ in an open set $R$ while $g(b), X^{s}(b), Y^{i s}(b)$ are of class $C^{\prime \prime}$ on $B$. Under the added assumption that $P(x, y, a)$ is Lipschitzian in $y$ and $a$, the indirect method of Hestenes is used to prove that the necessary conditions for relative minima of the problem above, strengthened in the usual manner, yield a set of sufficient conditions. This problem differs from that of Pontryagin in the choice of $(x, y, a)$ to lie in an open set.

Definitions and Notation. The arc $C$ will be denoted by

$$
C: b, y(x), a(x)
$$

and the minimizing arc will be called $C_{0}$. A set of parameters $\beta^{\circ}$ and functions $\eta^{i}(x), \alpha^{h}(x)$ is called a variation $\gamma$ and denoted by

$$
\gamma: \beta, \eta(x), \alpha(x)
$$

if $\eta^{i}(x)$ are continuous and $\dot{\eta}^{i}(x), \alpha^{h}(x)$ are in $L_{2}$ on $x^{1} \leqq x \leqq x^{2}$. The variation $\gamma$ is differentially admissible if

$$
\dot{\eta}=P_{y} \eta^{j}+P_{a^{h}} \alpha^{h}
$$

along $C_{0}$ for almost all $x$ on $x^{1} \leqq x \leqq x^{2}$. Repeated indices indicate summation. It is admissible if in addition to being differentially admissible 
it also satisfies the variational end conditions

$$
\eta^{i}\left(x^{s}\right)=\left\{Y_{\rho}^{i s}-\dot{y}^{i}\left(x^{s}\right) X_{\rho}^{s}\right\} \beta^{\rho}=C_{\rho}^{i s} \beta^{\rho} \quad(s=1,2)
$$

where the subscript $\rho$ denotes the derivative with respect to $b^{\rho}$.

2. Condition $S$. An admissible are

$$
C_{0}: b_{0}, y_{0}(x), a_{0}(x)
$$

will be said to satisfy condition $S$ if the following are true.

(a) $a_{0}(x)$ is continuous on $X^{1}\left(b_{0}\right) \leqq x \leqq X^{2}\left(b_{0}\right)$.

(b) $C_{0}$ satisfies the first necessary conditions, i.e., the Euler equations,

$$
\dot{z}^{i}(x)=-H_{y^{i}}, \dot{y}^{i}(x)=H_{z^{i}}, H_{a^{h}}=0
$$

and the transversality condition

$$
g_{\rho}-\left[H\left(x_{0}^{s}\right) X_{\rho}^{s}-z^{i}\left(x_{0}^{s}\right) Y_{\rho}^{i s}\right]_{s=1}^{s=2}=0
$$

with $z^{i}(x)$ being continuous and having continuous derivatives on a neighborhood of $C_{0}$. The symbol $\left[f\left(x^{s}\right)\right]_{s=1}^{s=2}$ means $f\left(x^{2}\right)-f\left(x^{1}\right)$.

(c) $C_{0}$ is nonsingular, i.e., the determinant $\left|H_{a^{h} a^{k}}\right|$ is nonzero along $C_{0}$ where

$$
H(x, y, \alpha, z)=z^{i}(x) P^{i}(x, y, a)-f(x, y, \alpha) .
$$

(d) $C_{0}$ with $z^{i}(x)$ satisfies the strengthened condition $I I_{N}$ of Weierstrass, $E_{H}(x, y, p, q, z) \geqq 0$ whenever $(x, y, p, z)$ is near those on $C_{0}$ and $(x, y, p) \neq(x, y, q)$ in $R$. The $E$-function is given by

$$
\begin{aligned}
E_{H}(x, y, p, q, z)= & -H(x, y, q, z)+H(x, y, p, z) \\
& +\left(q^{h}-p^{h}\right) H_{p h}(x, y, p, z)
\end{aligned}
$$

(e) For every nonnull admissible variation $\gamma$, the second variation $I_{2}(\gamma)$ along $C_{0}$ is greater than zero where

$$
\begin{aligned}
& I_{2}(\gamma)=\left\{g_{\rho \sigma}-\left[H X_{\rho \sigma}^{s}-z^{i} Y_{\rho \sigma}^{i s}\right.\right. \\
&\left.\left.\quad+\left\{H_{x}-\dot{y}^{i} H_{y^{i}}\right\} X_{\rho}^{s} X_{\sigma}^{s}+H_{y^{i}}\left(Y_{\rho}^{i s} X_{\sigma}^{s}+Y_{\sigma}^{i s} X_{\rho}^{s}\right)\right]_{s=1}^{s=2}\right\} \beta^{\rho} \beta^{\sigma} \\
&-\int_{x^{1}}^{x^{2}} 2 \omega(x, \eta, \alpha) d x \\
& 2 \omega(x, \eta, \alpha)=H_{y^{i} y} \eta^{i} \eta^{j}+2 H_{y^{i} a^{h}} \eta^{i} \alpha^{h}+H_{a^{h} a^{k}} \alpha^{h} \alpha^{k}
\end{aligned}
$$

(f) There is a neighborhood of $C_{0}$ in $x y$-space such that

$$
|P(x, y, a)-P(x, Y, A)|<c\left\{|y-Y|^{2}+|a-A|^{2}\right\}^{1 / 2}, c>0
$$

holds for all elements $(x, y, a),(x, Y, A)$ of $R$ which have $(x, y)$ in that neighborhood. 
Unless otherwise specified it will be assumed that the are denoted by $C_{0}$ will satisfy condition $S$. The principal theorem of this paper can now be stated and its proof will be given in $\S 7$, using the results of the intervening sections.

THEOREM 2.1. Let $C_{0}$ be an admissible arc on $x^{1} \leqq x \leqq x^{2}$ satisfying condition $S$. There is a neighborhood $N$ of $C_{0}$ in $b y$-space such that $I(C)>I\left(C_{0}\right)$ for all admissible arcs $C$ with $(b, y)$ in $N$ and $(x, y, a)$ in $R$.

For future use it is convenient to state a theorem of Hestenes [8, Theorem 5.1] as

THEOREM 2.2. Let $C_{0}$ be a nonsingular admissible minimizing arc satisfying condition $I I_{N}$. There is a neighborhood $N_{0}$ of $C_{0}$ in b y a-space and a constant $h>0$ such that

$$
E_{H}(x, y, p, q, z) \geqq h l(q-p)
$$

for $(x, y, p)$ in $N_{0}$ and $(x, y, q)$ in $R$ where

$$
l(q-p)=\sqrt{1+|q-p|^{2}}-1
$$

and $|q-p|=$ the length of the vector $q-p$.

3. $I^{*}(C)$. Let $C_{0}$ be a nonsingular minimizing arc and define

$$
\begin{aligned}
E_{H}^{*}(C) & =\int_{x^{1}}^{x^{2}} E_{H}(C) d x \\
& =-\int_{x^{1}}^{x^{2}}\left\{-H(a)+H\left(a_{0}\right)+\left(a^{h}-a_{0}^{h}\right) H_{a^{h}}\left(a_{0}\right)\right\} d x
\end{aligned}
$$

where the missing arguments are $(x, y(x), z(x))$. Choose a function $I^{*}(C)$ so that

$$
I(C)=I^{*}(C)+E_{H}^{*}(C) .
$$

It follows from the definitions of $I(C)$ and $E_{H}^{*}(C)$ that

$$
\begin{aligned}
I^{*}(C)= & g(b)+\left[z^{i}\left(x^{s}\right) y^{i}\left(x^{s}\right)\right]_{s=1}^{s=2} \\
& -\int_{X^{1}(b)}^{X^{2}(b)}\left\{\dot{z}^{i}(x) y^{i}(x)+H\left(x, y, a_{0}, z\right)+\left\{a^{h}-a_{0}^{h}\right\} H_{a^{h}}\left(x, y, a_{0}, z\right)\right\} d x .
\end{aligned}
$$

Since $E_{H}^{*}\left(C_{0}\right)=0$,

$$
I(C)-I\left(C_{0}\right)=I^{*}(C)-I^{*}\left(C_{0}\right)+E_{H}^{*}(C) .
$$

From the definition of $I^{*}(C)$, 


$$
\begin{aligned}
I^{*}(C)-I^{*}\left(C_{0}\right)= & \left\{g(b)-g\left(b_{0}\right)\right\} \\
& +\left[z^{i}\left(x^{s}\right) y^{i}\left(x^{s}\right)-z^{i}\left(x_{0}^{s}\right) y_{0}^{i}\left(x_{0}^{s}\right)\right]_{s=1}^{s=2} \\
& -\int_{X^{1}(b)}^{X^{2}(b)}\left\{\dot{z}^{i}\left\{y^{i}-y_{0}^{i}\right\}+H(y)\right. \\
& \left.-H\left(y_{0}\right)+\left\{a^{h}-a_{0}^{h}\right\} H_{a^{h}}(y)\right\} d x \\
& -\int_{X^{2}\left(b_{0}\right)}^{X^{2}(b)}\left\{\dot{z}^{i} y_{0}^{i}+H\left(y_{0}\right)\right\} d x \\
& \quad+\int_{X^{1}\left(b_{0}\right)}^{X^{1}(b)}\left\{\dot{z}^{i} y_{0}^{i}+H\left(y_{0}\right)\right\} d x
\end{aligned}
$$

where the missing arguments in $H$ are $\left(x, a_{0}, z\right)$. The following result can now be proved.

THEOREM 3.1. Let $C_{0}$ be a nonsingular admissible minimizing arc satisfying condition $I I_{N}$. For every $\varepsilon>0$ there exists a constant $\delta>0$ and a neighborhood $F$ of $C_{0}$ in by-space such that

$$
\left|I^{*}(C)-I^{*}\left(C_{0}\right)\right|<\varepsilon\left\{1+E_{H}^{*}(C)\right\},
$$

for every admissible arc $C$ in $F$ whose endpoints are in a $\delta$-neighborhood of these on $C_{0}$.

Given $\varepsilon>0, \delta$ and a neighborhood $N_{1}$ of $C_{0}$ in $b y$-space can be chosen such that from equation (3.1),

$$
\left|I^{*}(C)-I^{*}\left(C_{0}\right)\right|<\left|\int_{X^{1}(b)}^{X^{2}(b)}\left\{a^{h}-a_{0}^{h}\right\} H_{g^{h}}\left(x, y, a_{0}, z\right) d x\right|+\frac{\varepsilon}{2}
$$

for all $\operatorname{arcs} C$ with $(b, y)$ in $N_{1}$. Since $H_{a^{h}}\left(x, y_{0}, a_{0}, z\right)=0$, it follows that for $\varepsilon>0$ a neighborhood $N_{2}$ of $C_{0}$ in $b y$-space can be chosen so that

$$
\left|H_{a^{h}}\left(x, y, a_{0}, z\right)\right|<\varepsilon_{1}
$$

for all $\operatorname{arcs} C$ with $(b, y)$ in $N_{2}$. From Theorem 2.2,

$$
E_{H}(C) \geqq h l(q-p)>h\left\{\left|a-a_{0}\right|-1\right\}
$$

and

$$
\left|a-a_{0}\right| \leqq \frac{1}{h}\left\{E_{H}(C)+h\right\} \text {. }
$$

This together with inequality (3.3) yields

$$
\begin{aligned}
\left|\int_{X^{1}}^{X^{2}}\left\{a^{h}-a_{0}^{h}\right\} H_{a^{h}}\left(x, y, a_{0}, z\right) d x\right| & <\varepsilon_{1} \int_{X^{1}}^{x^{2}}\left|a-a_{0}\right| d x \\
& <\frac{\varepsilon_{1}}{h}\left\{E_{H}^{*}(C)+h\left(x^{2}-x^{1}\right)\right\} .
\end{aligned}
$$


Choose $\varepsilon_{1}$ such that $\varepsilon_{1}\left(x^{2}-x^{1}\right)<\varepsilon / 2$ and $\varepsilon_{1} / h<\varepsilon$. If in addition $F$ is taken to be the smaller of the neighborhoods $N_{1}$ and $N_{2}$, the theorem follows readily from inequalities (3.2) and (3.4).

THEOREM 3.2. Given a constant $\sigma>0$ there are positive constants $\delta, \rho$ and $a$ neighborhood $F$ of $C_{0}$ in $b y$-space such that for every admissible arc $C$ in $F$ satisfying theorem $3.1, I(C)>I\left(C_{0}\right)-\sigma$. If $E_{H}^{*}(C) \leqq \rho$, then $I(C)<I\left(C_{0}\right)+\sigma$. If $E_{H}^{*}(C) \geqq 2 \sigma$, then $I(C)>$ $I\left(C_{0}\right)+\sigma$.

The definition of $I(C)$ and Theorem 3.1 yield

$$
-\varepsilon+\{1-\varepsilon\} E_{H}^{*}(C)<I(C)-I\left(C_{0}\right)<\varepsilon+\{1+\varepsilon\} E_{H}^{*}(C)
$$

for all admissible $\operatorname{arcs} C$ with $(b, y)$ in $F$. The theorem follows immediately from the proper choice of $\varepsilon$ and $\rho$.

4. Extension of the arcs $C_{0}$ and $C$. We shall extend the arcs $C_{0}, C$ to lie on a fixed interval $e^{1} \leqq x \leqq e^{2}$ containing $X^{1}\left(b_{0}\right) \leqq x \leqq X^{2}\left(b_{0}\right)$ and $X^{1}(b) \leqq x \leqq X^{2}(b)$. The equation

$$
H_{a^{h}}(x, y, a, z)=0
$$

has a solution $y=y_{0}(x), a=a_{0}(x)$ corresponding to the minimizing arc $C_{0}$. By the nonsingularity of $C_{0}$, there is a solution $a=a(x, y, z)$ of equation (4.1) which is continuous and has continuous derivatives in a neighborhood of $C_{0}$. Further, on $X^{1}\left(b_{0}\right) \leqq x \leqq X^{2}\left(b_{0}\right), a\left(x, y_{0}, z\right)=a_{0}(x)$. By an imbedding theorem [2, pp. 196] the equations

$$
\begin{aligned}
& \dot{y}=H_{z}(x, y, a(x, y, z)) \\
& \dot{z}=-H_{y}(x, y, a(x, y, z))
\end{aligned}
$$

have a solution $y=\bar{y}(x), z=\bar{z}(x)$ on $e^{1} \leqq x \leqq e^{2}$ such that $e^{1}<X^{1}\left(b_{0}\right)<$ $X^{2}\left(b_{0}\right)<e^{2}$ and $\bar{y}(x)=y_{0}(x), \bar{z}(x)=z_{0}(x)$ on $X^{1}\left(b_{0}\right) \leqq x \leqq X^{2}\left(b_{0}\right)$. The $\operatorname{arc} \bar{C}_{0}$,

$$
\bar{C}_{0}: b_{0}, \bar{y}(x), \bar{a}(x)=a(x, \bar{y}(x), \bar{z}(x))
$$

coincides with $C_{0}$ on $x^{1} \leqq x \leqq x^{2}$, is defined on the larger interval $e^{1} \leqq x \leqq e^{2}$ and is therefore an extension of the arc $C_{0}$. Since this extension is unique, the extended are will be denoted by $C_{0}$,

$$
C_{0}: b_{0}, y_{0}(x)=\bar{y}(x), a_{0}(x)=\bar{a}(x) \text {. }
$$

If an admissible arc $C$ lies in a sufficiently small neighborhood of $C_{0}$ then $e^{1} \leqq X^{1}(b)<X^{2}(b) \leqq e^{2}$ and the arc $C$ may be extended uniquely to the interval $e^{1} \leqq x \leqq e^{2}$ by requiring that $a(x)=a_{0}(x)$ where it is undefined and that $\dot{y}=P(x, y, a(x))$ also holds on the extension. The extended arc will also be denoted by $C$. 
This method of extension will be used throughout the rest of the paper. In the formulas for $I(C)$ and $I^{*}(C)$ it will be understood that the integrals will be evaluated on the interval $x^{1} \leqq x \leqq x^{2}$ and not on the extended interval. An exception to this convention is made in the formula for $K\left(C, C_{0}\right)$ which is discussed in the next session.

5. The function $K\left(C, C_{0}\right)$. To measure the deviation of comparison ares from the minimizing arc, we shall define a function $K\left(C, C_{0}\right)$ where $C, C_{0}$ are the unique extensions of admissible arcs given in the last section as

$$
K\left(C, C_{0}\right)=\left|b-b_{0}\right|^{2}+\max _{e^{1} \leqq x \leqq e^{2}}\left|y(x)-y_{0}(x)\right|^{2}+\int_{e^{1}}^{e^{2}} l\left(\alpha-a_{0}\right) d x
$$

with

$$
l\left(a-a_{0}\right)=\sqrt{1+\left|a-a_{0}\right|^{2}}-1 .
$$

Since $a(x)=a_{0}(x)$ on the extension,

$$
\int_{e^{1}}^{e^{2}} l\left(a-a_{0}\right) d x=\int_{x^{1}}^{x^{2}} l\left(a-a_{0}\right) d x
$$

and $E_{H}(C)$ is not changed by extending the interval.

THEOREM 5.1. Let $C, C_{0}$ be extensions to $e^{1} \leqq x \leqq e^{2}$ of an admissible arc and a nonsingular minimizing arc respectively. For every $\varepsilon>0$ there is a b y-neighborhood of $C_{0}$ such that $K\left(C, C_{0}\right)<\varepsilon$ for all arcs $C$ in that neighborhood satisfying $E_{H}^{*}(C)<\varepsilon / 2$.

By Theorem 2.2 and the hypothesis,

$$
\frac{\varepsilon}{2}>E_{H}^{*}(C)>h \int_{x^{1}}^{x^{2}} l\left(a-a_{0}\right) d x .
$$

Choose a neighborhood of $C_{0}$ in $b y$-space such that

$$
\left|b-b_{0}\right|^{2}+\max _{e^{1} \leqq x \leqq e^{2}}\left|y(x)-y_{0}(x)\right|^{2}<\frac{(2 h-1) \varepsilon}{2 h} .
$$

In that neighborhood,

$$
K\left(C, C_{0}\right)<\frac{(2 h-1) \varepsilon}{2 h}+\frac{\varepsilon}{2 h}=\varepsilon
$$

and the theorem is proved.

THEOREM 5.2. Let $C_{q}$ be the extension of an admissible arc and let the sequence $\left\{C_{q}\right\}$ of such extended arcs have the property that given 
a neighborhbood $F$ of $C_{0}$ in $b y$-space there is an integer $q_{0}$ such that $C_{q}$ is in $F$ for $q>q_{0}$. If $\lim \sup _{q=\infty} I\left(C_{q}\right) \leqq I\left(C_{0}\right)$, then $\lim _{q=\infty} K\left(C_{q}, C_{0}\right)=0$.

If $F$ is the neighborhood in Theorem 3.2 and $E_{H}^{*}\left(C_{q}\right) \geqq 2 \sigma$ for $q>q_{0}, \sigma>0, I\left(C_{q}\right)>I\left(C_{0}\right)+\sigma$ which contradicts the hypothesis that $\lim \sup _{q=\infty} I\left(C_{q}\right) \leqq I\left(C_{0}\right)$. Hence, $E_{H}^{*}\left(C_{q}\right) \leqq 2 \sigma<\varepsilon / 4$. Theorem 5.1 asserts that $K\left(C_{q}, C_{0}\right)<\varepsilon$ for arbitrary $\varepsilon>0$ and the theorem is proved.

TheOREM 5.3. The sequence of arcs $\left\{C_{q}\right\}$ in Theorem 5.2 has the property that $\left\{b_{q}\right\}$ converges to $b_{0},\left\{y_{q}(x)\right\}$ converges uniformly to $y_{0}(x)$ and $\left\{a_{q}(x)\right\}$ converges almost uniformly in subsequence to $a_{0}(x)$.

Since $\lim _{q=\infty} K\left(C_{q}, C_{0}\right)=0$, it follows that

$$
\begin{gathered}
\lim _{q=\infty}\left|b_{q}-b_{0}\right|^{2}=0, \\
\lim _{q=\infty} \max _{e^{1} \leqq x \leqq e^{2}}\left|y_{q}(x)-y_{0}(x)\right|^{2}=0,
\end{gathered}
$$

and

$$
\lim _{q=\infty} \int_{e^{1}}^{e^{2}} l\left(a_{q}-a_{0}\right) d x=0 .
$$

The first two of these equalities give the convergence properties of the sequences $\left\{b_{q}\right\}$ and $\left\{y_{q}(x)\right\}$ respectively. Suppose now that there is a subset $S$ of $e^{1} \leqq x \leqq e^{2}$ of positive measure, $m(S)>0$, such that for any integer $q_{0}$ there is a $q>q_{0}$ for which $\left|a_{q}(x)-a_{0}(x)\right|>\sigma>0$ for all $x$ in $S$. Then, since $l\left(a_{q}-a_{0}\right) \geqq 0$ for all $q$, it follows that

$$
\int_{e^{1}}^{e^{2}} l\left(a_{q}-a_{0}\right) d x \geqq \int_{S} l\left(a_{q}-a_{0}\right) d x>\left\{\sqrt{1+\sigma^{2}}-1\right\} m(S)>0
$$

for infinitely many $q$ 's. This contradicts equation (5.1) and the sequence $\left\{a_{q}(x)\right\}$ must converge in measure to $a_{0}(x)$ on $e^{1} \leqq x \leqq e^{2}$. There is then a subsequence, call it $\left\{a_{q}(x)\right\}$, which converges almost uniformly to $a_{0}(x)$ on $e^{1} \leqq x \leqq e^{2}$ and the theorem is proved.

THEOREM 5.4. Let $\left\{C_{q}\right\}$ be a sequence of extended arcs having the convergence properties of the last theorem. Given a constant $\rho>0$ there is a constant $\delta>0$ and an integer $q_{0}$ such that if $M$ is a subset of $e^{1} \leqq x \leqq e^{2}$ of measure at most $\delta$ and $q \geqq q_{0}$ then

$$
0 \leqq \int_{M} l_{q}(x) d x<\rho
$$

where $l_{q}(x)=l\left(a_{q}-a_{0}\right)+2=1+\sqrt{1+\left|a_{q}-a_{0}\right|^{2}}$.

By the definition of $l_{q}(x)$, 


$$
\int_{M} l_{q}(x) d x \leqq 2 \delta+\int_{M} l\left(\alpha_{q}-a_{0}\right) d x .
$$

If $q_{0}$ is chosen so that $K\left(C_{q}, C_{0}\right)<\rho / 2$ for all $q>q_{0}$ and $\delta$ is chosen to be $\rho / 4$, the right side of the desired inequality is proved. The proof is completed by noting that $l_{q}(x) \geqq 0$. We have just proved that $\int_{M} l_{q}(x) d x$ is an absolutely continuous function of $M$ uniformly with respect to $q$.

6. Variations $\gamma_{q}, \gamma_{0}$. Let $k_{q}$ be the positive square root of $K\left(C_{q}, C_{0}\right)$ and define a variation $\gamma_{q}$ as follows.

$$
\gamma_{q}: \beta_{q}=\frac{b_{q}-b_{0}}{k_{q}}, \quad \eta_{q}(x)=\frac{y_{q}(x)-y_{0}(x)}{k_{q}}, \quad \alpha_{q}(x)=\frac{a_{q}(x)-a_{0}(x)}{k_{q}} .
$$

For a sequence of ares $C_{q}$ with the property that $\lim _{q=\infty} K\left(C_{q}, C_{0}\right)=0$ it will be shown that the sequence of variations $\left\{\gamma_{q}\right\}$ converges in subsequence to a variation $\gamma_{0}$ which is admissible on $x^{1} \leqq x \leqq x^{2}$. From the definitions of $\gamma_{q}$ and $K\left(C_{q}, C_{0}\right)$ it follows that

$$
\left|\beta_{q}\right|^{2}+\max _{e^{1} \leqq x \leqq e^{2}}\left|\eta_{q}(x)\right|^{2}+\int_{e^{1}}^{e^{2}} \frac{\left|\alpha_{q}(x)\right|^{2}}{l_{q}(x)} d x=1 .
$$

Since each term is nonnegative.

$$
\begin{gathered}
\left|\beta_{q}\right|^{2} \leqq 1, \\
\max _{e^{1} \leqq x \leqq e^{2}}\left|\eta_{q}(x)\right|^{2} \leqq 1,
\end{gathered}
$$

and

$$
\int_{e^{1}}^{e^{2}} \frac{\left|\alpha_{q}(x)\right|^{2}}{l_{q}(x)} d x \leqq 1
$$

Using these inequalities we shall obtain several theorems, the first of which is

THEOREM 6.1. Let $\left\{C_{q}\right\}$ be a sequence of extended arcs for which $\lim _{q=\infty} K\left(C_{q}, C_{0}\right)=0$ and $\beta_{q}=\left(b_{q}-b_{0}\right) / k_{q}$. The sequence $\left\{\beta_{q}\right\}$ converges in subsequence to a parameter $\beta_{0}$.

This follows immediately from inequality (6.2) and the BolzanoWeierstrass theorem.

THEOREM 6.2. Let $\left\{C_{q}\right\}$ be the sequence of arcs in the previous theorem and $\alpha_{q}(x)=\left(a_{q}(x)-a_{0}(x)\right) / k_{q}$. There is a function $\alpha_{0}(x)$ in $L_{2}$ on $e^{1} \leqq x \leqq e^{2}$ such that the sequence $\left\{\alpha_{q}(x)\right\}$ converges weakly in 
subsequence to $\alpha_{0}(x)$ in $L_{2}$ on every measurable set $M$ on which $a_{q}(x)$ converges uniformly to $a_{0}$. Moreover, for every bounded integrable function $g(x)$,

$$
\lim _{q=\infty} \int_{e^{1}}^{e^{2}} g(x) \alpha_{q}(x) d x=\int_{e^{1}}^{e^{2}} g(x) \alpha_{0}(x) d x .
$$

From inequality (6.4) and the inequality of Schwarz,

$$
\left|\int_{M} \alpha_{q}(x) d x\right|^{2} \leqq \int_{M} \frac{\left|\alpha_{q}(x)\right|^{2}}{l_{q}(x)} d x \int_{M} l_{q}(x) d x \leqq \int_{M} l_{q}(x) d x
$$

for all measurable subsets $M$ of $e^{1} \leqq x \leqq e^{2}$. Hence

$$
\lim _{m(M)=0} \int_{M} \alpha_{q}(x) d x=0
$$

by Theorem 5.4 and $\int_{M} \alpha_{q}(x) d x$ is absolutely continuous in $M$ uniformly with respect to $q$. In addition, equation (5.1) and the definition of $l_{q}(x)$ imply that there is an integer $q_{0}$ such that for $q>q_{0}, \int_{e^{1}}^{e^{2}} l_{q}(x)$ is bounded. Hence $\int_{e^{1}}^{e^{2}}\left|\alpha_{q}(x)\right| d x$ is bounded. Banach [1] proved that there is an integrable function $\alpha_{0}(x)$ such that the sequence $\left\{\alpha_{q}(x)\right\}$ satisfies equation (6.5) for all bounded integrable functions $g(x)$.

Now let $M$ be a subset of $e^{1} \leqq x \leqq e^{2}$ on which $\left\{a_{q}(x)\right\}$ converges uniformly to $a_{0}(x)$. For $x$ in $M$ there is an integer $q_{1}$ such that for $q>q_{1}, l_{q}(x)<3$. Thus $\int_{M}\left|\alpha_{q}(x)\right|^{2} d x<3$ for all $q>q_{1}$. Banach [1, p. 130] showed that for a sequence of functions $\left\{\alpha_{q}(x)\right\}$ in $L_{2}$ satisfying this last inequality, there is a function $\alpha_{0}(x)$ in $L_{2}$ to which $\left\{\alpha_{q}(x)\right\}$ converges weakly in $L_{2}$ in subsequence on $M$. Consequently,

$$
3 \geqq \liminf _{q=\infty} \int_{M}\left|\alpha_{q}(x)\right|^{2} d x \geqq \int_{M}\left|\alpha_{0}(x)\right|^{2} d x .
$$

Since this holds for every set $M$ as above, we have $\int_{e^{1}}^{e^{2}}\left|\alpha_{0}(x)\right|^{2} d x \leqq 3$ and $\alpha_{0}(x)$ is in $L_{2}$ on $e^{1} \leqq x \leqq e^{2}$. The theorem is thus proved.

THEOREM 6.3. Let $\left\{C_{q}\right\}$ be the sequence of arcs in the previous theorem and let $\eta_{q}(x)=\left(y_{q}(x)-y_{0}(x)\right) / k_{q}$. There exists a function $\eta_{0}(x)$ whose derivative $\dot{\eta}_{0}(x)$ is in $L_{2}$ such that the sequence $\left\{\eta_{q}(x)\right\}$ converges uniformly to $\eta_{0}(x)$ on $e^{1} \leqq x \leqq e^{2}$ and $\left\{\dot{\eta}_{q}(x)\right\}$ converges weakly in $L_{2}$ to $\dot{\eta}_{0}(x)$ on every measurable set $M$ on which $\left\{a_{q}(x)\right\}$ converges uniformly to $a_{0}(x)$. Moreover,

$$
\lim _{q=\infty} \int_{e^{1}}^{e^{2}} g(x) \dot{\eta}_{q}(x) d x=\int_{e^{1}}^{e^{2}} g(x) \dot{\eta}_{0}(x) d x
$$

for every bounded measurable function $g$. 
Applying the Lipschitz condition of condition $S$ to equation (6.1), we get

$$
\left|\beta_{q}\right|^{2}+\max _{e \leqq x \leqq e^{2}}\left|\eta_{q}(x)\right|^{2}+\frac{1}{c^{2}} \int_{e^{1}}^{e^{2}} \frac{\left|\dot{\eta}_{q}(x)\right|^{2}}{l_{q}(x)} d x \leqq 1+\int_{e^{1}}^{e^{2}} \frac{\left|\eta_{q}(x)\right|^{2}}{l_{q}(x)} d x .
$$

Since $\max _{e^{1} \leqq x \leqq e^{2}}\left|\eta_{q}(x)\right|^{2} \leqq 1$ and $l_{q}(x) \geqq 2$,

$$
\int_{e^{1}}^{e^{2}} \frac{\left|\eta_{q}(x)\right|^{2}}{l_{q}(x)} d x<\frac{1}{2} \int_{e^{1}}^{e^{2}} d x=\frac{1}{2}\left(e^{2}-e^{1}\right)=c_{1},
$$

a constant. Hence,

$$
\left|\beta_{q}\right|^{2}+\max _{e^{1} \leqq x \leqq e^{2}}\left|\eta_{q}(x)\right|^{2}+\frac{1}{c^{2}} \int_{e^{1}}^{e^{2}} \frac{\left|\dot{\eta}_{q}(x)\right|^{2}}{l_{q}(x)} d x \leqq 1+c_{1} .
$$

By an argument similar to that for the sequence $\left\{\alpha_{q}(x)\right\}$ it follows that there is a function $\dot{\eta}_{0}(x)$ in $L_{2}$ to which the sequence $\left\{\dot{\eta}_{q}(x)\right\}$ converges weakly. Hence,

$$
\lim _{q=\infty} \int_{e^{1}}^{x} \dot{\eta}_{q}(t) d t=\int_{e^{1}}^{x} \dot{\eta}_{0}(t) d t
$$

uniformly on $e^{1} \leqq x \leqq e^{2}$. Let

$$
\eta_{0}^{i}(x)=C_{\rho}^{i 1} \beta_{0}^{\rho}+\int_{x^{1}}^{x} \dot{\eta}_{0}(t) d t .
$$

Since $\lim _{q=\infty} \eta_{q}\left(X^{1}\left(b_{q}\right)\right)=\eta_{0}\left(x^{1}\right)$, it follows from (6.6) that the sequence $\left\{\eta_{q}(x)\right\}$ converges uniformly to $\eta_{0}(x)$ on $e^{1} \leqq x \leqq e^{2}$ and the theorem is proved.

THEOREM 6.4. Let $\left\{C_{q}\right\}$ be the sequence of extended arcs for which $\lim _{q=\infty} K\left(C_{q}, C_{0}\right)=0$ and define the variation $\gamma_{q}$ as above. The sequence of variations $\left\{\gamma_{q}\right\}$ converges in subsequence to a variation $\gamma_{0}$ which is admissible on $x^{1} \leqq x \leqq x^{2}$.

Let $\gamma_{0}$ consist of the parameters $\beta_{0}$ and the functions $\eta_{0}(x), \alpha_{0}(x)$ of the preceding three theorems. That $\gamma_{0}$ is a variation follows directly from the definition of a variation and the properties of $\beta_{0}, \eta_{0}(x)$, and $\alpha_{0}(x)$. The variation $\gamma_{0}$ will be admissible if it is differentially admissible and satisfies the endpoint equations in $\S 1$. Let $M_{\delta}$ be a subset of $x^{1} \leqq x \leqq x^{2}$ on which $\left\{a_{q}(x)\right\}$ converges uniformly to $a_{0}(x)$ and whose complement relative to $x^{1} \leqq x \leqq x^{2}$ has measure less than $\delta, \delta>0$. By Taylor's theorem,

$$
\dot{y}_{q}-\dot{y}_{0}=P_{y j}\left\{y_{q}^{j}-y_{0}^{j}\right\}+P_{a^{h}}\left\{a_{q}^{h}-a_{0}^{h}\right\}+R_{q},
$$

the arguments of $P_{y^{j}}, P_{a^{h}}$ being $\left(x, y_{0}, a_{0}\right)$ and 


$$
\left|R_{q}\right| \leqq \varepsilon_{q}\left\{\left|y_{q}-y_{0}\right|+\left|a_{q}-a_{0}\right|\right\}
$$

on $M$ where $\varepsilon_{q} \rightarrow 0$ as $q \rightarrow \infty$. Then

$$
\lim _{q=\infty} \int_{M_{\delta}} \dot{\eta}_{q}(x) d x=\lim _{q=\infty} \int_{M_{\delta}}\left\{P_{y^{j} j} \eta_{q}^{j}+P_{a^{k}} \alpha_{q}^{h}\right\} d x+\lim _{q=\infty} \int_{M_{\delta}} \frac{R_{q}}{k_{q}} d x .
$$

Since the last integral on the right is bounded and $\varepsilon_{q} \rightarrow 0$ as $q \rightarrow \infty$, it follows from Theorems 6.2 and 6.3 that

$$
\int_{M \delta} \dot{\eta}_{0}(x) d x=\int_{M_{\delta}}\left\{P_{y^{j}} \eta_{0}^{j}+P_{a h} \alpha_{0}^{h}\right\} d x
$$

and $\gamma_{0}$ is differentially admissible. The endpoint conditions on an admissible arc yield

$$
y_{q}^{i}\left(x^{s}\right)-y_{0}^{i}\left(x_{0}^{s}\right)=Y^{i s}\left(b_{q}\right)-Y^{i s}\left(b_{0}\right) .
$$

Expressing the left side as $y_{q}\left(x^{s}\right)-y_{0}\left(x^{s}\right)+y_{0}\left(x^{s}\right)-y_{0}\left(x_{0}^{s}\right)$ and dividing by $k_{q}$ we get

$$
\eta_{q}^{i}\left(x^{s}\right)+\dot{y}_{0}^{i}\left(x_{0}^{\prime s}\right) X_{\rho}^{s}\left(b_{0}^{\prime}\right) \beta_{q}^{\rho}=Y_{\rho}^{i s}\left(b_{0}^{\prime}\right) \beta_{q}^{\rho}
$$

where

$$
\begin{aligned}
x_{0}^{\prime s} & =x_{0}^{s}+\theta_{1}\left(x^{s}-x_{0}^{s}\right), 0<\theta_{1}<1 \\
b_{0}^{\prime} & =b_{0}+\theta_{2}\left(b_{q}-b_{0}\right), 0<\theta_{2}<1 .
\end{aligned}
$$

When $q \rightarrow \infty$,

$$
\eta_{0}^{i}\left(x_{0}^{s}\right)=\left\{Y_{\rho}^{i s}-\dot{y}_{0}^{i} X_{\rho}^{s}\right\} \beta_{0}^{\rho}=C_{\rho}^{i s} \beta_{0}^{\rho}
$$

and $\gamma_{0}$ is admissible.

7. Proof of the sufficiency theorem. Two theorems involving $I^{*}\left(C_{q}\right)$ and $E_{H}^{*}\left(C_{q}\right)$ will be proved, then they will be used to obtain a proof of the sufficiency theorem of $\S 2$.

THEOREM 7.1. Let $C_{0}$ be an admissible arc on $x^{1} \leqq x \leqq x^{2}$ satisfying condition $S$. If for any integer $q$ there is an admissible arc $C_{q} \neq C_{0}$ in the $1 / q$-neighborhood of $C_{0}$ such that $I\left(C_{q}\right) \leqq I\left(C_{0}\right)$ then

$$
\lim _{q=\infty} \frac{I^{*}\left(C_{q}\right)-I^{*}\left(C_{0}\right)}{k_{q}^{2}}=\frac{1}{2} I_{2}\left(\gamma_{0}\right)+\frac{1}{2} \int_{x^{1}}^{x^{2}} H_{a^{h} a^{k}} \alpha_{0}^{h} \alpha_{0}^{k} d x \text {. }
$$

Applying Taylor's theorem to the right side of equation (3.1) for $I^{*}(C)-I^{*}\left(C_{0}\right)$ and dividing by $k_{q}^{2}$ we get equations (7.1) to (7.4)

$$
\frac{g\left(b_{q}\right)-g\left(b_{0}\right)}{k_{q}^{2}}=\frac{1}{k_{q}} g_{\rho} \beta_{q}^{\rho}+\frac{1}{2} g_{\rho \sigma} \beta_{q}^{\sigma}+R_{1 q}
$$


where $\left|R_{1 q}\right|<\varepsilon_{1 q}\left|\beta_{q}\right|^{2}$ and $\lim _{q=\infty} \varepsilon_{1 q}=0$. The derivatives are evaluated at $b=b_{0}$.

$$
\begin{aligned}
& \frac{z^{i}\left(x^{s}\right) Y^{i s}\left(b_{q}\right)-z^{i}\left(x_{0}^{s}\right) Y^{i s}\left(b_{0}\right)}{k_{q}^{2}}=\frac{1}{k_{q}}\left[\dot{z}^{i} Y^{i s} X^{s}+z^{i} Y_{\rho}^{i s}\right]_{s=1}^{s=1} \beta_{q}^{\rho} \\
& +\frac{1}{2}\left[\ddot{z}^{i} Y^{i s} X_{\rho}^{s} X_{\sigma}^{s}+\right. \\
& \dot{z}^{i}\left\{Y_{\sigma}^{2 s} X_{\rho}^{s}+Y_{\rho}^{i s} X_{\sigma}^{s}\right\} \\
& \left.+\dot{z}^{i} Y^{\imath s} X_{\rho \sigma}^{s}+z^{i} Y_{\rho \sigma}^{i s}\right]_{s=1}^{s=1} \beta_{q}^{s} \beta_{q}^{\sigma}+R_{2 q}
\end{aligned}
$$

where $\left|R_{2 q}\right|<\varepsilon_{2 q}\left|\beta_{q}\right|^{2}$ and $\lim _{q=\infty} \varepsilon_{2 q}=0$. Again the derivatives are evaluated at $b=b_{0}$.

$$
\begin{aligned}
\frac{1}{k_{q}^{2}} \int_{x^{1}}^{x^{2}}\left\{\dot{z}^{i}\left(y_{q}^{2}-y_{0}^{i}\right)+\right. & \left\{H\left(x, y_{q}, a_{0}, z\right)-H\left(x, y_{0}, a_{0}, z\right)\right\} \\
& \left.+\left(a^{h}-a_{0}^{h}\right) H_{a}\left(x, y_{q}, a_{0}, z\right)\right\} d x \\
= & \int_{x^{1}}^{x^{2}}\left\{\frac{1}{2} H_{\left.y^{i} y\right\lrcorner} \eta_{q}^{i} \eta_{q}^{j}+H_{y^{2} q} h \eta_{q}^{i} \alpha_{q}^{h}\right\} d x+\int_{x^{1}}^{x^{2}} R_{3 q} d x
\end{aligned}
$$

where $\left|R_{3 q}\right|<\varepsilon_{3 q}\left|\eta_{q}\right|^{2}$ and $\lim _{q=\infty} \varepsilon_{3 q}=0$. The derivatives $H_{y^{i} y^{j}}, H_{y^{i} a^{k}}$ are evaluated along $C_{0}$.

$$
\begin{aligned}
& \frac{1}{k_{q}^{2}} \int_{x^{1}\left(b_{0}\right)}^{x^{1}(b)}\left\{\dot{z}^{i} y_{0}^{i}+H\left(x, y_{0}, a_{0}, z\right)\right\} d x \\
&=\frac{1}{k_{q}}\left\{\dot{z}^{i} y_{0}^{i}+H\left(x, y_{0}, a_{0}, z\right)\right\} X_{\rho}^{1} \beta_{q}^{\rho} \\
&+\frac{1}{2}\left\{\ddot{z}^{i} y_{0}^{i}+H_{x}+H_{q h} \dot{\alpha}_{0}^{h}+H_{z^{2}} \dot{z}^{i}\right\} X_{\rho}^{1} X_{\sigma}^{1} \beta_{q}^{\rho} \beta_{q}^{\sigma} \\
&+\frac{1}{2}\left\{\dot{z}^{i} y_{0}^{i}+H\right\} X_{\rho \sigma}^{1} \beta_{q}^{p} \beta_{q}^{\sigma}+R_{4 q}
\end{aligned}
$$

where $\left|R_{4 q}\right|<\varepsilon_{4 q}\left|\beta_{q}\right|^{2}$ and $\lim _{q=\infty} \varepsilon_{4 q}=0$. All the terms on the right are evaluated along $C_{0}$ at $x=X^{1}\left(b_{0}\right)$. A result similar to this also holds for the integral remaining in the expression for $\left(I^{*}\left(C_{q}\right)-I^{*}\left(C_{0}\right)\right) / k_{q}^{2}$ with $R_{5 q}$ as the error in place of $R_{4 q}$. The definition of $R_{3 q}$ and the boundedness of $\left|\eta_{q}\right|^{2}$ yield the fact that $\lim _{q=\infty} \int_{x^{1}}^{x^{2}} R_{3 q} d x=0$. Substituting equations (7.1) to (7.4) into equation (3.1), applying condition $S$ and a theorem of Hestenes [7, Lemma 6.3] we get the desired result.

THEOREM 7.2. Let $C_{0}$ be an admissible are satisfying condition S. Let $\left\{C_{q}\right\}$ be admissible arcs related to $C_{0}$ as described in the last theorem and chosen so that the corresponding variation $\gamma_{q}$ defined previously converge to a variation $\gamma_{0}$ as described. Then

$$
\liminf _{q=\infty} \frac{E_{H}^{*}\left(C_{q}\right)}{k_{q}^{2}}+\frac{1}{2} \int_{x^{1}\left(b_{0}\right)}^{x^{2}\left(b_{0}\right)} H_{a^{h} a^{k}} \alpha_{0}^{h} \alpha_{0}^{k} d x \geqq 0 .
$$


For large $q, E_{H}\left(C_{q}\right)>0$ for $C_{q} \neq C_{0}$. Applying Taylor's theorem to $E_{B}\left(C_{q}\right)$ it follows that

$$
\frac{E_{H}^{*}\left(C_{q}\right)}{k_{q}^{2}} \geqq-\frac{1}{2} \int_{M} H_{a^{h} a^{k}}\left(x, y_{q}, a_{0}, z\right) \alpha_{q}^{h} \alpha_{q}^{k} d x+\int_{M} R_{6 q} d x
$$

where $M$ is a subset of $x^{1} \leqq x \leqq x^{2}$ on which $\left\{a_{q}(x)\right\}$ converges uniformly to $a_{0}(x)$. Since $\left|R_{6 q}\right|<\varepsilon_{6 q}\left|\alpha_{q}\right|^{2}$ and $\lim _{q=\infty} \varepsilon_{6 q}=0$ it follows from the boundedness of $\int_{x^{1}}^{x^{2}}\left|\alpha_{q}\right|^{2} d x$ that $\lim _{q=\infty} \int_{\mu} R_{6 q} d x=0$. Now

$$
\begin{aligned}
& -\frac{1}{2} \int_{M} H_{a^{h^{k}}}\left(x, y_{q}, a_{0}, z\right) \alpha_{q}^{h} \alpha_{q}^{k} d x \\
= & -\frac{1}{2} \int_{M} H_{a^{h_{a}}{ }^{k}}\left(x, y_{0}, a_{0}, z\right) \alpha_{0}^{h} \alpha_{0}^{k} d x \\
& -\frac{1}{2} \int_{M}\left\{H_{a^{h_{a}} a^{k}}\left(x, y_{q}, a_{0}, z\right)-H_{a^{h_{a}}{ }^{k}}\left(x, y_{0}, a_{0}, z\right)\right\} \alpha_{q}^{h} \alpha_{q}^{k} d x \\
& -\frac{1}{2} \int_{M} H_{a^{h^{k}} a^{k}}\left(x, y_{0}, a_{0}, z\right)\left\{\alpha_{q}^{h} \alpha_{q}^{k}-\alpha_{0}^{h} \alpha_{0}^{k}\right\} d x .
\end{aligned}
$$

From the continuity of $H_{a^{h} a^{k}}$ and the boundedness of $\int_{x^{1}}^{x^{2}}\left|\alpha_{q}\right|^{2} d x$ we get

$$
\lim _{q=\infty} \int_{\mathcal{M}}\left\{H_{a^{h^{k}}}\left(x, y_{q}, a_{0}, z\right)-H_{a^{h^{k}}}\left(x, y_{0}, a_{0}, z\right)\right\} \alpha_{q}^{h} \alpha_{q}^{k} d x=0 .
$$

The last integral in equation (7.7) can be written as

$$
\begin{aligned}
& \int_{M} H_{a^{h_{a} k}} \alpha_{q}^{h} \alpha_{q}^{k} d x=\int_{M} H_{a^{h_{a}}{ }^{k}}\left\{\alpha_{q}^{h}-\alpha_{0}^{h}\right\}\left\{\alpha_{q}^{k}-\alpha_{0}^{k}\right\} d x \\
& +\int_{M} H_{a^{h_{a} k}}\left\{\alpha_{q}^{h} \alpha_{0}^{k}+\alpha_{0}^{h} \alpha_{q}^{k}\right\} d x-\int_{M} H_{a^{h_{a} k}{ }^{k}} \alpha_{0}^{h} \alpha_{0}^{k} d x .
\end{aligned}
$$

Since $\left\{\alpha_{q}(x)\right\}$ converges weakly to $\alpha_{0}(x)$ on $M$,

$$
\begin{aligned}
\liminf _{q=\infty} & \int_{M}-\frac{1}{2} H_{a^{h} a^{k}} \alpha_{q}^{h} \alpha_{q}^{k} d x=-\frac{1}{2} \int_{M} H_{a^{h} a^{k}} \alpha_{0}^{h} \alpha_{0}^{k} d x \\
& +\liminf _{q=\infty} \int_{M}-\frac{1}{2} H_{a^{h} a^{k}}\left\{\alpha_{q}^{h}-\alpha_{0}^{h}\right\}\left\{\alpha_{q}^{k}-\alpha_{0}^{k}\right\} d x .
\end{aligned}
$$

Therefore, from (7.6), (7.7) and (7.8),

$$
\begin{aligned}
\liminf _{q=\infty} & \frac{E_{H}^{*}\left(C_{q}\right)}{k_{2}^{q}}+\frac{1}{2} \int_{M} H_{a^{h} a^{k}} \alpha_{0}^{h} \alpha_{0}^{k} d x \\
& \geqq \liminf _{q=\infty} \int_{M}-\frac{1}{2} H_{a^{h} a^{k}}\left\{\alpha_{q}^{h}-\alpha_{0}^{h}\right\}\left\{\alpha_{q}^{k}-\alpha_{0}^{k}\right\} d x .
\end{aligned}
$$

Since $C_{0}$ satisfies condition $\mathrm{II}_{N}$ with multipliers $z^{i}(x)$ it also satisfies the strengthened condition of Clebsch, 


$$
H_{a^{h} a_{k}} \pi^{h} \pi^{k} \leqq 0
$$

in a neighborhood of $C_{0}$ for all $(\pi) \neq(0)$. Hence the last integral in (7.9) is nonnegative and the theorem is proved for every subset $M$ on which $\left\{a_{q}(x)\right\}$ converges uniformly to $a_{0}(x)$. Let $M_{1}$ be the complement of $M$ on $x^{1} \leqq x \leqq x^{2}$. Then

$$
\int_{x^{1}}^{x_{j}} H_{a^{h^{k}} a^{k}} \alpha_{0}^{h} \alpha_{0}^{k} d x=\int_{M} H_{a^{h^{k}}} \alpha_{0}^{h} \alpha_{0}^{k} d x+\int_{\mathbb{M}_{1}} H_{a^{h_{a}}} \alpha_{0}^{h} \alpha_{0}^{k} d x .
$$

Since the integrand $H_{a^{h_{a}{ }^{k}}} \alpha_{0}^{h} \alpha_{0}^{k}$ is integrable on $x^{1} \leqq x \leqq x^{2}$, the last integral of the preceding equation must go to zero as the measure of $M_{1}$ tends to zero. Thus the theorem is proved over $x^{1} \leqq x \leqq x^{2}$. We now turn to the proof of Theorem 2.1. Suppose it is false. For any integer $q$ there is an admissible are $C_{q} \neq C_{0}$ in the $1 / q$-neighborhood of $C_{0}$ such that $I\left(C_{q}\right) \leqq I\left(C_{0}\right)$. From equation (3.2) and Theorem 7.1,

$$
0 \geqq I_{2}\left(\gamma_{0}\right)+\frac{1}{2} \int_{x^{1}}^{x^{2}} H_{a^{h_{a}}{ }^{k}} \alpha_{0}^{h} \alpha_{0}^{k} d x+\liminf _{q=\infty} \frac{E_{H}^{*}\left(C_{q}\right)}{k_{q}^{2}}
$$

which implies, by virtue of Theorem 7.2, that $I_{2}\left(\gamma_{0}\right) \leqq 0$. Statement (e) of condition $S$ requires that $\gamma_{0}$ must be null. Consequently $I_{2}\left(\gamma_{0}\right)=0$ and

$$
\int_{x^{1}}^{x^{2}} H_{a^{h} a^{k}} \alpha_{0}^{h} \alpha_{0}^{k} d x=0 .
$$

By Theorem 2.2 and the inequality (7.10),

$$
0 \geqq \liminf _{q=\infty} \frac{E_{H}^{*}\left(C_{q}\right)}{k_{q}^{2}}=h \liminf _{q=\infty} \int^{x^{2}} \frac{\left|\alpha_{q}\right|^{2}}{l_{q}(x)} d x
$$

which is impossible because of equation (6.1). Hence $\gamma_{0} \neq 0$ and the assumption that $I\left(C_{q}\right) \leqq I\left(C_{0}\right)$ is false. This proves the sufficiency theorem.

\section{REFERENCES}

1. S. Banach, Theorie des Operations Lineaires, Warsaw, 1932.

2. G. A. Bliss, Lectures on the Calculus of Variations, University of Chicago Press, 1945.

3. M. R. Hestenes, A General Problem in the Calculus of Variations with Applications to Paths of Least Time, RAND Research Memorandum, RM-100, 1950.

4. The Weierstrass E-function in the calculus of variation, Trans. Amer. Math. Soc. 60 (1946), 51-71.

5. — Theorem of Lindeberg in the calculus of variations, Trans. Amer. Soc. 60 (1946), 72-92.

6. Sufficient conditions for the isoperimetric problem of Bolza in the calculus of variation, Trans. Amer. Math. Soc. 60 (1946), 93-118. 
7. - An Indirect sufficiency proof for the problem of Bolza in nonparametric form, Trans. Amer. Math. Soc. 62 (1947), 509-535.

8. _ - Sufficient conditions for multiple integral problems in the calculus of variations, Amer. J. Math. 70 (1948), 239-276.

9. E. J. McShane, Sufficient conditions for a weak relative minimum in the problem of Bolza. Trans. Amer. Math. Soc. 52 (1942), 344-379. 



\section{PACIFIC JOURNAL OF MATHEMATICS}

\section{EDITORS}

\author{
H. SAMELSON \\ Stanford University \\ Stanford, California \\ R. M. BLUMENTHAL \\ University of Washington \\ Seattle, Washington 98105
}

\author{
*J. DUGUNDJI \\ University of Southern California \\ Los Angeles, California 90007
}

RICHARD ARENS

University of California Los Angeles, California 90024

\section{ASSOCIATE EDITORS}
E. F. BECKENBACH
B. H. NEUMANN
F. WOLF
K. YoSIDA

\section{SUPPORTING INSTITUTIONS}

\author{
UNIVERSITY OF BRITISH COLUMBIA \\ CALIFORNIA INSTITUTE OF TECHNOLOGY \\ UNIVERSITY OF CALIFORNIA \\ MONTANA STATE UNIVERSITY \\ UNIVERSITY OF NEVADA \\ NEW MEXICO STATE UNIVERSITY \\ OREGON STATE UNIVERSITY \\ UNIVERSITY OF OREGON \\ OSAKA UNIVERSITY \\ UNIVERSITY OF SOUTHERN CALIFORNIA
}

\author{
STANFORD UNIVERSITY \\ UNIVERSITY OF TOKYO \\ UNIVERSITY OF UTAH \\ WASHINGTON STATE UNIVERSITY \\ UNIVERSITY OF WASHINGTON \\ * * * * \\ AMERICAN MATHEMATICAL SOCIETY \\ CHEVRON RESEARCH CORPORATION \\ TRW SYSTEMS \\ NAVAL ORDNANCE TEST STATION
}

Mathematical papers intended for publication in the Pacific Journal of Mathematics should be typewritten (double spaced). The first paragraph or two must be capable of being used separately as a synopsis of the entire paper. It should not contain references to the bibliography. Manuscripts may be sent to any one of the four editors. All other communications to the editors should be addressed to the managing editor, Richard Arens at the University of California, Los Angeles, California 90024.

50 reprints per author of each article are furnished free of charge; additional copies may be obtained at cost in multiples of 50 .

The Pacific Journal of Mathematics is published monthly. Effective with Volume 16 the price per volume (3 numbers) is $\$ 8.00$; single issues, $\$ 3.00$. Special price for current issues to individual faculty members of supporting institutions and to individual members of the American Mathematical Society: $\$ 4.00$ per volume; single issues $\$ 1.50$. Back numbers are available.

Subscriptions, orders for back numbers, and changes of address should be sent to Pacific Journal of Mathematics, 103 Highland Boulevard, Berkeley 8, California.

Printed at Kokusai Bunken Insatsusha (International Academic Printing Co., Ltd.), No. 6, 2-chome, Fujimi-cho, Chiyoda-ku, Tokyo, Japan.

\section{PUBLISHED BY PACIFIC JOURNAL OF MATHEMATICS, A NON-PROFIT CORPORATION}

The Supporting Institutions listed above contribute to the cost of publication of this Journal, but they are not owners or publishers and have no responsibility for its content or policies.

* Paul A. White, Acting Editor until J. Dugundji returns. 


\section{Pacific Journal of Mathematics}

\section{Vol. 17, No. 2 \\ February, 1966}

Henry A. Antosiewicz, Boundary value problems for nonlinear ordinary

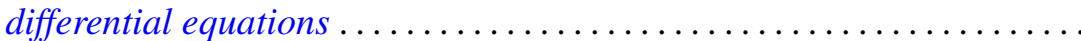

Bernard Werner Levinger and Richard Steven Varga, Minimal Gerschgorin sets. II ........................................

Paul Camion and Alan Jerome Hoffman, On the nonsingularity of complex matrices........................................ 211

J. Chidambaraswamy, Divisibility properties of certain factorials ........ 215

J. Chidambaraswamy, A problem complementary to a problem of Erdös .... 227

John Dauns, Chains of modules with completely reducible quotients ...... 235

Wallace E. Johnson, Existence of half-trajectories in prescribed regions and asymptotic orbital stability ............................ 243

Victor Klee, Paths on polyhedra. II . ........................ 249

Edwin Haena Mookini, Sufficient conditions for an optimal control problem in the calculus of variations ............................ 263

Zane Clinton Motteler, Existence theorems for certain quasi-linear elliptic equations........................................... 279

David Lewis Outcalt, Simple n-associative rings ............... 301

David Joseph Rodabaugh, Some new results on simple algebras ......... 311

Oscar S. Rothaus, Asymptotic properties of groups generation ........... 319

Ernest Edward Shult, Nilpotence of the commutator subgroup in groups admitting fixed point free operator groups .............

William Hall Sills, On absolutely continuous functions and the

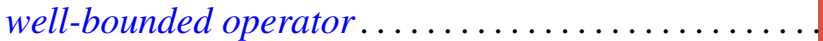

Joseph Gail Stampfli, Which weighted shifts are subnormal ..

Donald Reginald Traylor, Metrizability and completeness in normal Moore spaces 